\title{
Theoretical Update of Twist-3 Single-Spin Asymmetry in Semi-Inclusive DIS
}

\author{
Yuji Koike ${ }^{1}$ and Kazuhiro Tanaka ${ }^{2}$ \\ 1- Department of Physics, Niigata University, Ikarashi, Niigata 950-2181, Japan \\ 2- Department of Physics, Juntendo University, Inba, Chiba 270-1695, Japan
}

\begin{abstract}
We discuss the single-spin asymmetry in semi-inclusive DIS, $e p^{\uparrow} \rightarrow e \pi X$, based on the twist-3 mechanism in the collinear factorization relevant for the pion production with the large transverse-momentum. This updates our previous study by including, in particular, the contributions induced by the novel partonic subprocesses, and allows us to derive the entire formula for the corresponding single-spin asymmetry associated with a complete set of the twist-3 quark-gluon correlation functions in the transversely polarized nucleon. We discuss the correspondence with the results obtained by the transverse-momentum-dependent factorization relevant for the pion production with the low transverse-momentum.
\end{abstract}

The single transverse-spin asymmetry (SSA) in the semi-inclusive DIS (SIDIS), $e(\ell)+$ $p\left(p, S_{\perp}\right) \rightarrow e\left(\ell^{\prime}\right)+\pi\left(P_{h}\right)+X$, is observed as $T$-odd effect in the cross section for the scattering of transversely polarized nucleon with momentum $p$ and spin $S_{\perp}$, off unpolarized lepton with momentum $\ell$, producing a pion with momentum $P_{h}$ which is observed in the final state. Here, $q=\ell-\ell^{\prime}, Q^{2}=-q^{2}$, and $Q \gg \Lambda_{\mathrm{QCD}}$. The SSA can be observed also in $p p$ collisions, the pion production $p^{\uparrow} p \rightarrow \pi X$ [2], and the Drell-Yan and direct- $\gamma$ production, $p^{\uparrow} p \rightarrow \gamma^{(*)} X$ [3]. Similarly as these examples, the SSA in the SIDIS requires, (i) nonzero $P_{h \perp}$ originating from transverse motion of quark or gluon; (ii) nucleon helicity flip; and (iii) interaction beyond Born level to produce the interfering phase for the cross section. When $P_{h \perp} \ll Q$, all (i)-(iii) may be generated nonperturbatively from the T-odd, transverse-momentum-dependent (TMD) parton distribution/fragmentation functions such as the Sivers function (see [4, 5]). By contrast, for large $P_{h \perp} \gg \Lambda_{\mathrm{QCD}}$, (i) should come from perturbative mechanism as the recoil from the hard (unobserved) final-state partons, while nonperturbative effects can participate in the other two, (ii) and (iii), allowing us to obtain large SSA. This is realized with the twist-3 distribution/fragmentation functions in the collinear-factorization framework. In our recent paper [6], we have derived the corresponding factorization formula, in the leading-order perturbative QCD, for the SSA associated with the twist-3 distributions for the nucleon, and provided a practical procedure to calculate the relevant partonic hard parts manifesting their gauge invariance at the twist-3 level. The factorization formula in [6] implies, for the form of the twist-3 single-spin-dependent cross section as a function of the azimuthal angle $\phi_{h}$ of the final-state pion $\left(\Phi \equiv \phi_{h}-\phi_{S}\right)$ [7,

$$
\frac{d^{5} \sigma^{\mathrm{tw} 3}}{[d \omega]}=\left(\sigma_{1}^{\mathrm{tw} 3}+\sigma_{2}^{\mathrm{tw} 3} \cos \phi_{h}+\sigma_{3}^{\mathrm{tw} 3} \cos 2 \phi_{h}\right) \sin \Phi+\left(\sigma_{4}^{\mathrm{tw} 3} \sin \phi_{h}+\sigma_{5}^{\mathrm{tw} 3} \sin 2 \phi_{h}\right) \cos \Phi,
$$

in a frame where the 3-momenta $\vec{q}$ and $\vec{p}$ of the virtual photon and the transversely polarized nucleon are collinear along the $z$ axis; $\phi_{h}$ as well as the azimuthal angle $\phi_{S}$ of the nucleon's transverse-spin vector $S_{\perp}^{\mu}$ is measured from the lepton plane, and $[d \omega] \equiv d x_{b j} d Q^{2} d z_{f} d q_{T}^{2} d \phi_{h}$ denotes the differential element with the usual kinematical variables for the SIDIS, $x_{b j}=$ $Q^{2} /(2 p \cdot q), z_{f}=p \cdot P_{h} / p \cdot q$, and $q_{T}=P_{h \perp} / z_{f}$. In [6], we calculated the explicit form only 
for $\sigma_{1,2,3}^{\mathrm{tw} 3}$. In addition, a new type of partonic subprocesses that contribute to the twist- 3 mechanism has been pointed out in [8]. In this report we present the main features of the full result [9], taking into account these points as well as yet another type of the novel partonic subprocesses relevant to the twist-3 mechanism.

In the twist-3 mechanism relevant to (11), the nucleon helicity flip of (ii) above is provided by the quark-gluon correlation in its inside, which generates additional nonperturbative gluon as described by the nucleon matrix element of the three-body nonlocal operators on the lightcone, $\left\langle p S_{\perp}\left|\bar{\psi}(0) F^{\alpha+}(\xi n) \psi(\lambda n)\right| p S_{\perp}\right\rangle$, where $n^{2}=0, p \cdot n=1$, and $F^{\alpha \beta}$ is the gluon field strength tensor. The Fourier transform of this matrix element defines the two twist3 correlation functions, $G_{F}\left(x_{1}, x_{2}\right)$ and $\widetilde{G}_{F}\left(x_{1}, x_{2}\right)$, as the coefficients of the independent Lorentz structures, where $x_{1,2}$ denote the lightcone momentum fractions associated with the quark fields $\psi, \bar{\psi} ; G_{F}$ and $\widetilde{G}_{F}$ are symmetric and antisymmetric, respectively, under $x_{1} \leftrightarrow x_{2}$ and form a complete set to calculate (10) [10. The above additional gluon from the transversely polarized nucleon, carrying the momentum fraction $x_{2}-x_{1}$, participates into the partonic hard scattering, as in the diagrams in Figs. 1, 2 below. The coupling of this gluon allows an internal propagator in the partonic subprocess to be on-shell, and this produces the imaginary phase of (iii) above, as the pole contribution using $1 /\left(k^{2}+i \varepsilon\right)=\mathrm{P}\left(1 / k^{2}\right)-i \pi \delta\left(k^{2}\right)$. Depending on the resulting value of the momentum fractions $x_{1,2}$ at such poles, these poles are the soft-gluon pole (SGP) for $x_{1}=x_{2}$, the soft-fermion pole (SFP) for $x_{i}=0$, and the hard pole (HP) for $x_{i}=x_{b j}$, where $i=1$ or 2 . We note that the right four diagrams of Fig. 1 reduce to those representing the partonic hard part of the twist- 2 unpolarized cross section for the large- $P_{h \perp}$ pion production, $e p \rightarrow e \pi X$, if we remove the insertion of the additional gluon. In [6], we calculated all the partonic subprocesses associated with such coherent gluon. By contrast, the diagrams that are not associated with the coherent gluon, like the left four diagrams of Fig. 1 and the diagrams in Fig. 2, represent the novel partonic subprocesses [8, 9]; these contributions do not have straightforward counterpart in the TMD factorization approach, i.e., in the effects due to the intrinsic transverse motion of the valence Fock components in the nucleon. Now we summarize the main points for updating each of the SGP, SFP and HP contributions, compared with our previous results [6].

First of all, the SGP contribution occurs only from the diagrams involving the coherent gluon (see Figs. 8 and 10 of [6]). Thus, our task here is to calculate the SGP contribution to $\sigma_{4,5}^{\text {tw3 }}$ of (11) from the relevant diagrams, following the procedure given in [6]. Alternatively, one can use the master formula proved in [7] (see also [11]), yielding the full SGP contribution to (11) as

$$
\frac{d^{5} \sigma_{\mathrm{SGP}}^{\mathrm{tw} 3}}{[d \omega]}=\left.\frac{\pi M_{N}}{2 C_{F} z_{f}}\left(\sin \Phi \frac{\partial}{\partial q_{T}}+\frac{\cos \Phi}{q_{T}} \frac{\partial}{\partial \phi_{h}}\right) \frac{d^{5} \sigma_{\text {unpol }}^{\mathrm{tw} 2}}{[d \omega]}\right|_{f_{q}(x) \rightarrow G_{F}^{q}(x, x), D_{j}(z) \rightarrow \mathcal{C}_{j} z D_{j}(z)},
$$

where $C_{F}=\left(N_{c}^{2}-1\right) /\left(2 N_{c}\right), M_{N}$ is the nucleon mass, and $\left(\mathcal{N}_{q} \equiv \alpha_{e m}^{2} \alpha_{s} e_{q}^{2} /\left(8 \pi x_{b j}^{2} S_{e p}^{2} Q^{2}\right)\right)$

$$
\frac{d^{5} \sigma_{\text {unpol }}^{\mathrm{tw} 2}}{[d \omega]}=\sum_{j=q, g} \mathcal{N}_{q} \int \frac{d z}{z} \int \frac{d x}{x} D_{j}(z) f_{q}(x) \sum_{n=1}^{3} \widehat{\varrho}_{n}^{j q} \cos \left((n-1) \phi_{h}\right) \delta\left(\frac{q_{T}^{2}}{Q^{2}}-\left[\frac{1}{\widehat{x}}-1\right]\left[\frac{1}{\widehat{z}}-1\right]\right)
$$

is the twist- 2 unpolarized cross section for $e p \rightarrow e \pi X$, obeying the factorization formula with the twist-2, parton-fragmentation and quark-distribution functions, $D_{j}(z)$ and $f_{q}(x)$, for the pion and unpolarized nucleon, respectively. Here, $S_{e p}=(\ell+p)^{2}, \widehat{x}=x_{b j} / x, \widehat{z}=z_{f} / z$, and 

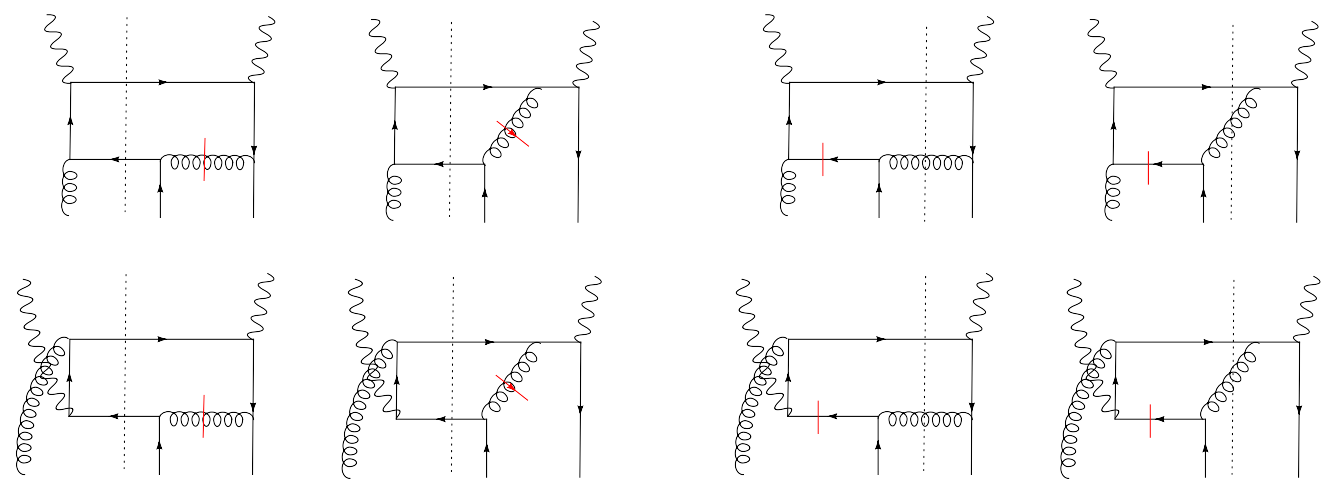

Figure 1: Diagrams for the SFP contribution to SSA in SIDIS. The bar denotes the pole contribution of the propagator. Either of the two partons going across the cut, the dotted line, fragments into the pion, while the other is unobserved. Mirror diagrams also contribute.

the sum over all quark and antiquark flavors, $q=u, \bar{u}, d, \bar{d}, \cdots$, is implicit for the index $q$; $\widehat{\varrho}_{n}^{j q}$ denote the partonic hard parts and read $\widehat{\varrho}_{1}^{j q}=\left(1+\cosh ^{2} \psi\right) \widehat{\sigma}_{1}^{j q}-2 \widehat{\sigma}_{2}^{j q}, \widehat{\varrho}_{2}^{j q}=-\widehat{\sigma}_{3}^{j q} \sinh 2 \psi$, and $\widehat{\varrho}_{3}^{j q}=\widehat{\sigma}_{4}^{j q} \sinh ^{2} \psi$, where $\cosh \psi=2 x_{b j} S_{e p} / Q^{2}-1$, and $\widehat{\sigma}_{k}^{j q}$ are given in Eqs. (57), (59) of [6] as the functions of $\widehat{x}, \widehat{z}, q_{T}$, and $Q$. For the replacements indicated in (2), $\mathcal{C}_{q}=-1 /\left(2 N_{c}\right), \mathcal{C}_{g}=N_{c} / 2$, and $G_{F}^{q}(x, x)$ for the flavor $q$ arises at the SGP corresponding to $x_{1}=x_{2}$ while $\widetilde{G}_{F}^{q}(x, x)=0$ due to the symmetry property. The $\phi_{h}$-dependence of (3) resides in $\cos \left((n-1) \phi_{h}\right)$ only, so that $\sigma_{4}^{\mathrm{tw} 3}$ and $\sigma_{5}^{\mathrm{tw} 3}$ of (1) are generated by the angular derivative in (2) and are related to $\sigma_{2}^{\mathrm{tw} 3}$ and $\sigma_{3}^{\mathrm{tw} 3}$, respectively. However, $\sigma_{4,5}^{\mathrm{tw} 3}$ do not receive the derivative of the twist-3 distribution, $d G_{F}^{q}(x, x) / d x$, which contributes to $\sigma_{1,2,3}^{\mathrm{tw} 3}$ through the $q_{T}$-derivative in (2) (see Erratum of [7] for the explicit formula obtained from (2) ).

Second, the SFP corresponding to $x_{1}=0$ in the diagrams of Fig. 1, the right four diagrams involving the coherent gluon [6] and the others of new type [8], picks up $G_{F}^{q}(0, x)$ as well as $\widetilde{G}_{F}^{q}(0, x)$. As pointed out in [8, the contributions of these two types of diagrams cancel with each other when the final-state parton with the flavor $q$, represented by the upper horizontal line across the cut, fragments into the pion. Thus, the SFP contributes to $\sigma_{1, \cdots, 5}^{\mathrm{tw} 3}$ of (1) accompanying $D_{j}(z) G_{F}^{q}(0, x)$ or $D_{j}(z) \widetilde{G}_{F}^{q}(0, x)$ with $j=\bar{q}, g$, corresponding to the case where the lower horizontal line across the final-state cut in Fig. 1 represents the fragmentation function $D_{j}(z)$. Calculating these relevant diagrams with the procedure in [6], our result obeys a simple relation that, for each of $\sigma_{1, \cdots, 5}^{\mathrm{tw} 3}$ in (1), the partonic hard parts associated with $D_{j}(z) G_{F}^{q}(0, x)$ differ only in the overall sign between $j=\bar{q}$ and $j=g$, and similarly for those associated with $D_{j}(z) \widetilde{G}_{F}^{q}(0, x)$; this relation reflects the above-mentioned cancellation for the channel corresponding to $D_{q}(z) G_{F}^{q}(0, x), D_{q}(z) \widetilde{G}_{F}^{q}(0, x)$.

Third, the HP corresponding to $x_{1}=x_{b j}$ in the diagrams involving the coherent gluon (see Fig. 2 in [6]) can be evaluated according to [6], and picks up $D_{j}(z) G_{F}^{q}\left(x_{b j}, x\right)$ and $D_{j}(z) \widetilde{G}_{F}^{q}\left(x_{b j}, x\right)$ with $j=q, g$. Decomposing the result into the independent azimuthal structures as in (11), we find the new results for $\sigma_{4,5}^{\mathrm{tw} 3}$, in addition to $\sigma_{1,2,3}^{\mathrm{tw} 3}$ obtained in [6]. The results show, for each term associated with $D_{j}(z) G_{F}^{q}\left(x_{b j}, x\right), D_{j}(z) \widetilde{G}_{F}^{q}\left(x_{b j}, x\right)$, that $\sigma_{4}^{\mathrm{tw} 3}$ and $\sigma_{5}^{\mathrm{tw} 3}$ coincide with $\sigma_{2}^{\mathrm{tw} 3}$ and $\sigma_{3}^{\mathrm{tw} 3}$, respectively. However, in addition to those diagrams associated with the $q g$ final states, the partonic subprocesses with the $q \bar{q}$ final 

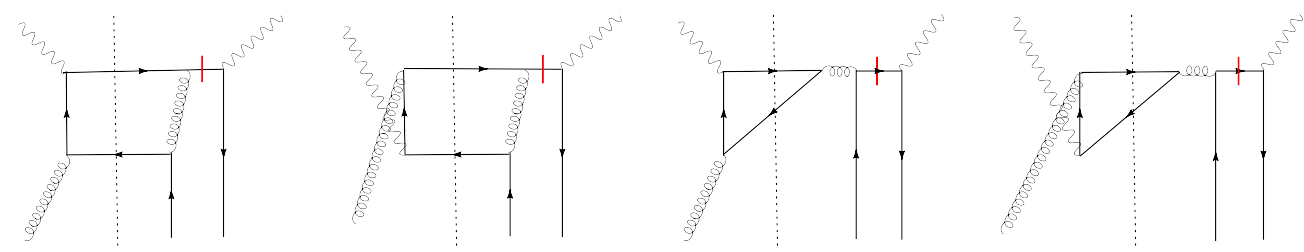

Figure 2: Same as Fig. 1, but for the new subprocesses that give rise to the HP contribution.

states can also receive the HP contribution at $x_{2}=x_{b j}$, as illustrated in Fig. 2. This would seem formally similar to the situation in the SFP contribution of Fig. 1, but, by contrast, the HP contribution of the novel diagrams of Fig. 2 does not have simple relation, for any fragmentation channel, to the above-mentioned HP contribution induced by the coherent gluon. Indeed, the HP in Fig. 2 picks up $D_{j}(z) G_{F}^{q}\left(x_{b j}, x_{b j}-x\right)$ and $D_{j}(z) \widetilde{G}_{F}^{q}\left(x_{b j}, x_{b j}-x\right)$ with $j=q, \bar{q}$, where $x_{b j}-x<0$ indicating that the nucleon absorbs a coherent $q \bar{q}$ pair in the RHS of the final-state cut in Fig. 2. Calculating these novel HP diagrams with the procedure of [6], we find $\sigma_{2}^{\mathrm{tw} 3, \mathrm{HP}} \neq \sigma_{4}^{\mathrm{tw} 3, \mathrm{HP}}$ and $\sigma_{3}^{\mathrm{tw} 3, \mathrm{HP}} \neq \sigma_{5}^{\mathrm{tw} 3, \mathrm{HP}}$ for the azimuthal structures of (1).

Collecting all the pole contributions discussed above, one obtains $\sigma_{n}^{\text {tw3 }}$ in the single-spindependent cross section (1), associated with the twist-3 functions $G_{F}$ and $\widetilde{G}_{F}$, as

$$
\begin{aligned}
& \sigma_{n}^{\mathrm{tw} 3}=\frac{-\pi M_{N} \mathcal{N}_{q}}{4} \sum_{j=q, \bar{q}, g} \int \frac{d z}{z} \int \frac{d x}{x} D_{j}(z)\left[\widehat{\sigma}_{D n}^{j q} x \frac{d G_{F}^{q}(x, x)}{d x}+\widehat{\sigma}_{G n}^{j q} G_{F}^{q}(x, x)+\widehat{\sigma}_{F n}^{j q} G_{F}^{q}(0, x)\right. \\
& \left.+\widehat{\sigma}_{H O n}^{j q} G_{F}^{q}\left(x_{b j}, x\right)+\widehat{\sigma}_{H N n}^{j q} G_{F}^{q}\left(x_{b j}, x_{b j}-x\right)\right] \delta\left(\frac{q_{T}^{2}}{Q^{2}}-\left[1-\frac{1}{\widehat{x}}\right]\left[1-\frac{1}{\widehat{z}}\right]\right)+\left[\widetilde{G}_{F} \text { terms }\right],(4)
\end{aligned}
$$

for $n=1,2,3,4,5$, where the partonic hard parts obey $\widehat{\sigma}_{D n}^{\bar{q} q}=\widehat{\sigma}_{G n}^{\bar{q} q}=\widehat{\sigma}_{F n}^{q q}=\widehat{\sigma}_{H O n}^{\bar{q} q}=$ $\widehat{\sigma}_{H N n}^{g q}=0, \widehat{\sigma}_{F n}^{\bar{q} q}=-\widehat{\sigma}_{F n}^{g q}, \widehat{\sigma}_{H O 2}^{j q}=\widehat{\sigma}_{H O 4}^{j q}$, and $\widehat{\sigma}_{H O 3}^{j q}=\widehat{\sigma}_{H O 5}^{j q}$, and " $\left[\widetilde{G}_{F}\right.$ terms $]$ " have the similar structure as the preceding terms except that the SGP contributions vanish $\left(\widetilde{G}_{F}(x, x)=0\right)$. The results (2), (3) further tell us that $\widehat{\sigma}_{D n}^{j q}=-\left[4 \mathcal{C}_{j} q_{T} \widehat{x} / C_{F} Q^{2}(1-\widehat{z})\right] \widehat{\varrho}_{n}^{j q}$ and $\widehat{\sigma}_{G n}^{j q}=-\left(4 \mathcal{C}_{j} q_{T} / C_{F} Q^{2}\right)\left\{\left(Q^{2} / \widehat{z}\right) \partial \widehat{\varrho}_{n}^{j q} / \partial q_{T}^{2}-[\widehat{x} /(1-\widehat{z})] \partial\left(\widehat{x} \widehat{\varrho}_{n}^{j q}\right) / \partial \widehat{x}\right\}$, for $n=1,2,3$, while $\widehat{\sigma}_{D n}^{j q}=0$ and $\widehat{\sigma}_{G n}^{j q}=\left(2^{n-3} \mathcal{C}_{j} / C_{F} q_{T} \widehat{z}\right) \widehat{\varrho}_{n-2}^{j q}$, for $n=4,5$. The explicit form of $\widehat{\sigma}_{F n}^{j q}, \widehat{\sigma}_{H O n}^{j q}, \widehat{\sigma}_{H N n}^{j q}$ and of the corresponding partonic hard parts for the "[ $\widetilde{G}_{F}$ terms $]$ " will be presented elsewhere [9]. We note that the delta function in (4) vanishes when $x<x_{b j}\left\{1+z_{f} q_{T}^{2} /\left[\left(1-z_{f}\right) Q^{2}\right]\right\}$ or $z<z_{f}\left\{1+x_{b j} q_{T}^{2} /\left[\left(1-x_{b j}\right) Q^{2}\right]\right\}$. We recast (11) into

$$
\begin{aligned}
\frac{d^{5} \sigma^{\mathrm{tw} 3}}{[d \omega]}= & \sin \left(\phi_{h}-\phi_{S}\right) F^{\sin \left(\phi_{h}-\phi_{S}\right)}+\sin \left(2 \phi_{h}-\phi_{S}\right) F^{\sin \left(2 \phi_{h}-\phi_{S}\right)}+\sin \phi_{S} F^{\sin \phi_{S}} \\
& +\sin \left(3 \phi_{h}-\phi_{S}\right) F^{\sin \left(3 \phi_{h}-\phi_{S}\right)}+\sin \left(\phi_{h}+\phi_{S}\right) F^{\sin \left(\phi_{h}+\phi_{S}\right)}
\end{aligned}
$$

with the structure functions for the independent azimuthal structures, $F^{\sin \left(\phi_{h}-\phi_{S}\right)}=\sigma_{1}^{\text {tw } 3}$, $F^{\sin \left(2 \phi_{h}-\phi_{S}\right)}=\left(\sigma_{2}^{\mathrm{tw} 3}+\sigma_{4}^{\mathrm{tw} 3}\right) / 2, F^{\sin \phi_{S}}=\left(\sigma_{4}^{\mathrm{tw} 3}-\sigma_{2}^{\mathrm{tw} 3}\right) / 2, F^{\sin \left(3 \phi_{h}-\phi_{S}\right)}=\left(\sigma_{3}^{\mathrm{tw} 3}+\sigma_{5}^{\mathrm{tw} 3}\right) / 2$, and $F^{\sin \left(\phi_{h}+\phi_{S}\right)}=\left(\sigma_{5}^{\mathrm{tw} 3}-\sigma_{3}^{\mathrm{tw} 3}\right) / 2$, which are all determined by the two independent twist-3 distributions of the nucleon as (44). By contrast, when the transverse momentum $P_{h \perp}(=$ $z_{f} q_{T}$ ) of the pion is low, the relevant single-spin-dependent cross section is calculated by the TMD factorization approach 4, and the corresponding five structure functions defined similarly as (5) are expressed by the (many) independent TMD distributions of the nucleon. 
Our results (4), (5) hold for $q_{T} \gg \Lambda_{\mathrm{QCD}}$, and their small- $q_{T}$ behavior with $q_{T} \ll Q$ allows us to make further contact to the high- $q_{T}$ behavior of the TMD factorization result with $\Lambda_{\mathrm{QCD}} \ll q_{T} \ll Q$. The corresponding small- $q_{T}$ behavior of (5) was studied in [12, using our previous result [6, 10] for $\sigma_{n}^{\mathrm{tw} 3}$. With our present update (4), we obtain the small- $q_{T}$ behavior, $F^{\sin \left(\phi_{h}-\phi_{S}\right)} \sim 1 / q_{T}^{3}, F^{\sin \left(2 \phi_{h}-\phi_{S}\right)} \sim 1 / q_{T}^{2}, F^{\sin \phi_{S}} \sim 1 / q_{T}^{2}, F^{\sin \left(3 \phi_{h}-\phi_{S}\right)} \sim 1 / q_{T}$, and $F^{\sin \left(\phi_{h}+\phi_{S}\right)} \sim 1 / q_{T}$, which are actually controlled by the SGP and HP contributions because the SFP contributions are suppressed by the powers of $q_{T}$ for all five structure functions. Although these leading power behaviors are formally same as those of the previous results 12 mentioned above, now the novel HP contribution from the diagrams in Fig. 2 also contributes to these leading-power terms, except for $F^{\sin \left(3 \phi_{h}-\phi_{S}\right)}$. In this connection, it has been shown in [5, 8 that the most dominant $\sim 1 / q_{T}^{3}$ term from $F^{\sin \left(\phi_{h}-\phi_{S}\right)}$ coincides with the high- $q_{T}$ behavior of the Sivers effect in the TMD factorization, where, however, the novel HP contribution was not taken into account. These facts call for clarifying the role of the novel HP contribution concerning the connection between the twist-3 and Sivers mechanism, as well as its quantitative relevance in the cross section (5). We also mention

that (5) receives another twist-3 effect associated with the twist-3 fragmentation function of the pion, $\widehat{E}_{F}\left(z_{1}, z_{2}\right)$, for which we need more study.

To summarize, we have discussed the twist-3 mechanism for the SSA in SIDIS, induced by the quark-gluon correlation inside the nucleon, and updated and completed the formula for the corresponding single-spin-dependent cross section. Our cross section is expressed by the five structure functions corresponding to independent azimuthal structures, and also includes the novel partonic subprocesses associated with the $q \bar{q}$ final states, as well as the previously known subprocesses associated with the $q g$ final states. The novel contributions are related to non-minimal parton configurations inside the nucleon, describing interference between scattering from a coherent $q \bar{q}$ pair and from a single gluon. We finally note that our twist-3 mechanism disappears for the inclusive DIS, because of cancellation of the final-state interactions for the unobserved final states [7, 9].

We thank F. Yuan and A. Metz for useful discussions.

\section{References}

[1] Slides: http: //indico. cern. ch/contributionDisplay $\cdot$ py? contribId=122\&sessionId=4\&conf $I d=53294$

[2] Y. Koike and T. Tomita, Phys. Lett. B675 181 (2009), and references therein.

[3] X. D. Ji, J. W. Qiu, W. Vogelsang, F. Yuan, Phys. Rev. D73 094017 (2006).

[4] A. Bacchetta, M. Diehl, K. Goeke, A. Metz, P. J. Mulders and M. Schlegel, JHEP 0702093 (2007).

[5] X. D. Ji, J. W. Qiu, W. Vogelsang, F. Yuan, Phys. Lett. B638 178 (2006).

[6] H. Eguchi, Y. Koike and K. Tanaka, Nucl. Phys. B763 193 (2007).

[7] Y. Koike and K. Tanaka, Phys. Lett. B646 232 (2007) [Erratum-ibid. B668 458 (2008)].

[8] Y. Koike, W. Vogelsang, F. Yuan, Phys. Lett. B659 878 (2008).

[9] Y. Koike and K. Tanaka, to be published.

[10] H. Eguchi, Y. Koike and K. Tanaka, Nucl. Phys. B752 1 (2006).

[11] Y. Koike and K. Tanaka, Phys. Rev. D76 011502 (2007).

[12] A. Bacchetta, D. Boer, M. Diehl and P. J. Mulders, JHEP 0808023 (2008). 